\title{
Histological complete response in advanced gastric cancer after 2 weeks of S-1 administration as neoadjuvant chemotherapy
}

\author{
Shusuke Mori ${ }^{1}$, Hirofumi Kishimoto ${ }^{1}$, Katsunori Tauchi ${ }^{1}$, and Kayoko Higuchi ${ }^{2}$ \\ ${ }^{1}$ Department of Surgery, Aizawa Hospital, 2-5-1 Honjou, Matsumoto, Nagano 390-8510, Japan \\ ${ }^{2}$ Department of Pathology, Aizawa Hospital, Nagano, Japan
}

\begin{abstract}
Single-agent or combined chemotherapy with the novel oral fluoropyrimidine anticancer drug, S-1 (TS-1), has been reported to be useful for the treatment of advanced gastric cancer. Here, we report a patient with advanced gastric cancer achieving a complete response (CR) after 2 weeks of administration of S-1 as neoadjuvant chemotherapy. A 78-year-old woman with epigastric pain was diagnosed as having advanced gastric cancer. S-1 was administered orally, at a dose of $50 \mathrm{mg}$ twice a day every day for $\mathbf{2}$ weeks, followed by a 2-week drugfree period. No obvious adverse reactions occurred. Subsequently, the patient underwent distal partial gastrectomy with D2 lymph node dissection. Pathological examination indicated no remnant signet-ring cells in the excised specimen, no lymph node metastasis, and unnatural fibrosis in one of the No. 3 lymph nodes. The neoadjuvant chemotherapy induced a CR according to the Japanese classification of gastric carcinoma.
\end{abstract}

Key words Advanced gastric cancer - Neoadjuvant chemotherapy $\cdot \mathrm{S}-1 \cdot$ Complete response

\section{Introduction}

Previous reports have suggested the usefulness of S-1 (1 $\mathrm{M}$ tegafur- $0.4 \mathrm{M}$ gimestat $1 \mathrm{M}$ otastat potassium) in the treatment of advanced gastric cancer. As it is effective when administered orally and has a low incidence of severe adverse effects [1,2], S-1 is expected to be suitable for outpatient neoadjuvant chemotherapy. In recent studies, patients have shown complete responses (CR) after neoadjuvant chemotherapy with S-1 alone or with combined therapy [3-6]. In each case, the patients took S-1 for 4 weeks or more, i.e., one course of the standard regimen, as their tumors were diagnosed as

Offprint requests to: $\mathrm{S}$. Mori

Received: September 13, 2005 / Accepted: December 27, 2005 unresectable. In cases evaluated as resectable, before we can offer neoadjuvant chemotherapy it is necessary to confirm the efficacy of S-1. The administration of neoadjuvant chemotherapy results in a delay before surgery, and the therapy may have little or no effect on primary or metastatic lesions. From the standpoint of cancer progression and adverse effects, it is best to keep the period of neoadjuvant chemotherapy as short as possible. We anticipate that shorter periods of neoadjuvant chemotherapy will be confirmed to be effective to reduce or cure gastric cancer.

\section{Case report}

A 78-year-old woman consulted her doctor because of epigastric pain. Endoscopy demonstrated an irregular depressed lesion in the lesser curvature of the antrum of the stomach (Fig. 1), and the biopsy revealed signet-ring cell carcinoma. Abdominal and thoracic computed tomography (CT) and positron emission tomography (PET) indicated that there were no metastases to the lymph nodes, liver, or other organs. A diagnosis of advanced gastric cancer was made (cT2, cN0, cM0, cStage IB according to the Japanese classification of gastric carcinoma, 2nd English edition [7]). We recommended that the patient undergo surgical resection as soon as possible, but she requested a delay of 1 month due to a memorial service. As this resulted in a delay of 1 month before the operation, we offered neoadjuvant chemotherapy, consisting of oral S-1. With informed consent, the patient $\left(148 \mathrm{~cm}, 46 \mathrm{~kg}, 1.36 \mathrm{~m}^{2}\right)$ took S-1 at a dose of $50 \mathrm{mg}$ twice a day for 2 weeks orally, followed by a drug-free period of 2 weeks. No adverse reactions were observed.

Distal partial gastrectomy and lymph node dissection (D2) were performed, with Billroth I reconstruction. Macroscopically, the excised specimen showed an irregular depressed lesion, measuring $30 \times 42 \mathrm{~mm}$, with 


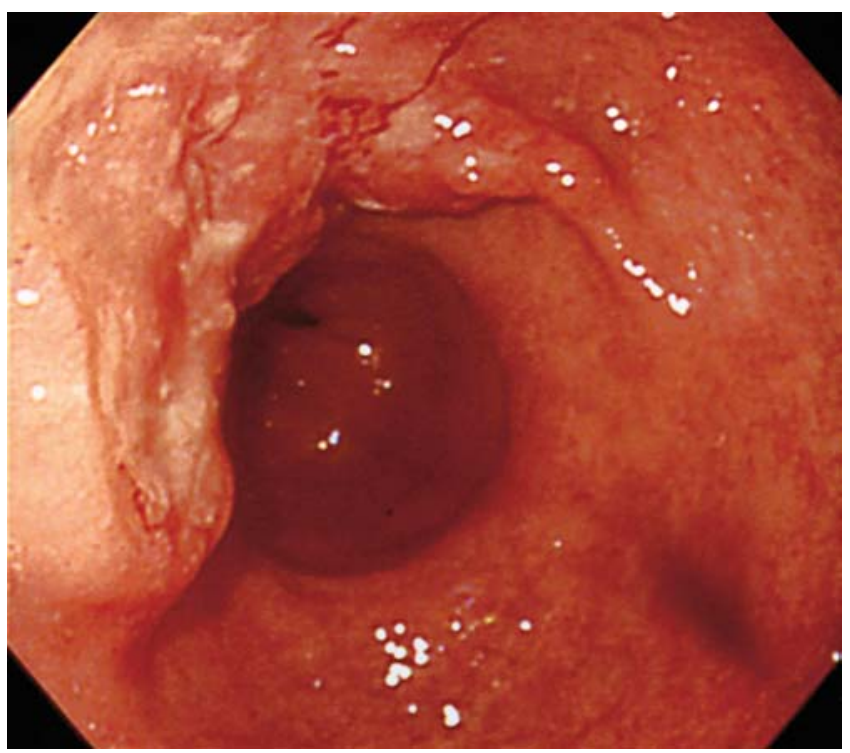

Fig. 1. Endoscopy showed an irregular depressed lesion in the lesser curvature of the antrum. The biopsy specimen showed signet-ring cell carcinoma. Together with the results of imaging studies, these studies led to a preoperative diagnosis of cT2, cN0, cM0, cStage IB

fibrosis through the wall of the stomach, in the lesser curvature of the antrum (Fig. 2). Microscopic findings indicated that the lesion was covered with regenerative mucosa, and it showed fibrosis and lymph follicle formation from the submucosa to the subserosa (Fig. 3A). Although negligible gland degeneration was seen in the mucosa, no signet-ring cell remnants were detected by immunofluorescence staining for CK22 (Fig. 3B). No lymph node metastasis was found pathologically, but one of the No. 3 lymph nodes showed unnatural fibrosis, suggesting degeneration of cancer cells (Fig. 3C). The patient was assessed as showing a complete response (CR) following neoadjuvant chemotherapy, corresponding to histological efficacy grade $3[8,9]$.

\section{Discussion}

Previous studies have shown S-1 to be an excellent oral anticancer drug for the treatment of advanced gastric cancer, and have suggested that this agent may be useful for neoadjuvant chemotherapy [3]. S-1 is an antitumor agent based on the biochemical modulation of a 5-fluorouracil (FU) derivative 1-(2-tetrahydrofuryl)5-fluorouracil (tegafur; FT) by 5-chloro-2,4dihydroxypyridine (gimestat; CDHP) and otastat potassium (Oxo) at a molar ratio of FT: $\mathrm{CDHP}$ : Oxo = 1:0.4:1. CDHP potentiates antitumor activity and Oxo reduces gastrointestinal toxicity, and both of these function as modulators [10]. Representative adverse reac-

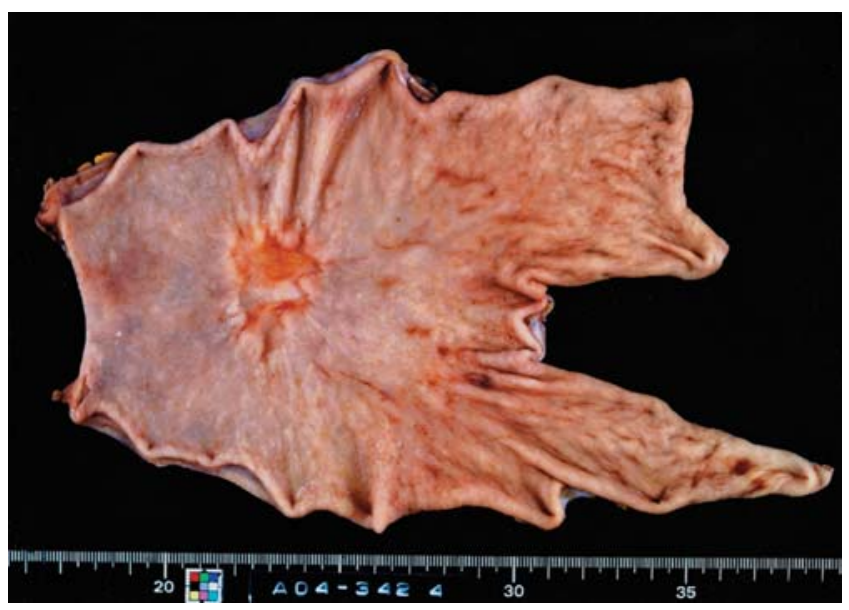

Fig. 2. Macroscopic findings of the excised specimen showed an irregular depressed lesion, measuring $30 \times 42 \mathrm{~mm}$, with fibrosis through the wall of the stomach, in the lesser curvature of the antrum

tions with S-1 include decreases in hematocrit level, leukopenia, granulocytopenia, diarrhea, malaise, and proteinuria [1]. A postmarketing survey suggested that toxicities were generally mild and that outpatient treatment with this agent would be feasible [11]. The present patient developed none of these adverse reactions during neoadjuvant chemotherapy or during the perioperative period. Two late phase II studies of S-1 in gastric cancer showed an overall response rate of $44.6 \%$ (45/101), with a $95 \%$ confidence interval (CI) of 35.962.3. Administration of S-1 alone showed efficacy comparable to that of combination therapy, with such agents as 5-FU+cisplatin (CDDP) or 5-FU+leucovorin $[1,2]$.

The preoperative diagnosis of the tumor in the present patient was advanced gastric carcinoma (cT2, cN0, cM0, cStage IB [7]). Therefore, we recommended that the patient undergo surgery as soon as possible, according to the guidelines for the treatment of gastric cancer [12]. Although there was a delay of 1 month prior to surgery, based on the patient's request, the efficacy of oral S-1 for only 2 weeks was remarkable, resulting in a postoperative diagnosis of a histological CR. Patients with advanced gastric cancer showing CR with the administration of S-1 alone as neoadjuvant chemotherapy have been reported, but in all such patients the course of S-1 treatment lasted for 4 weeks or more [3-6]. The standard regimen of S-1 is 4 weeks' administration followed by a 2-week drug-free period. A comparative study between the 4-week regimen and the 2-week regimen showed that the incidence of adverse reactions was lower in the 2 -week regimen group $(77 \%)$ than in the 4 -week regimen group (93\%), with little difference in efficacy [13]. 

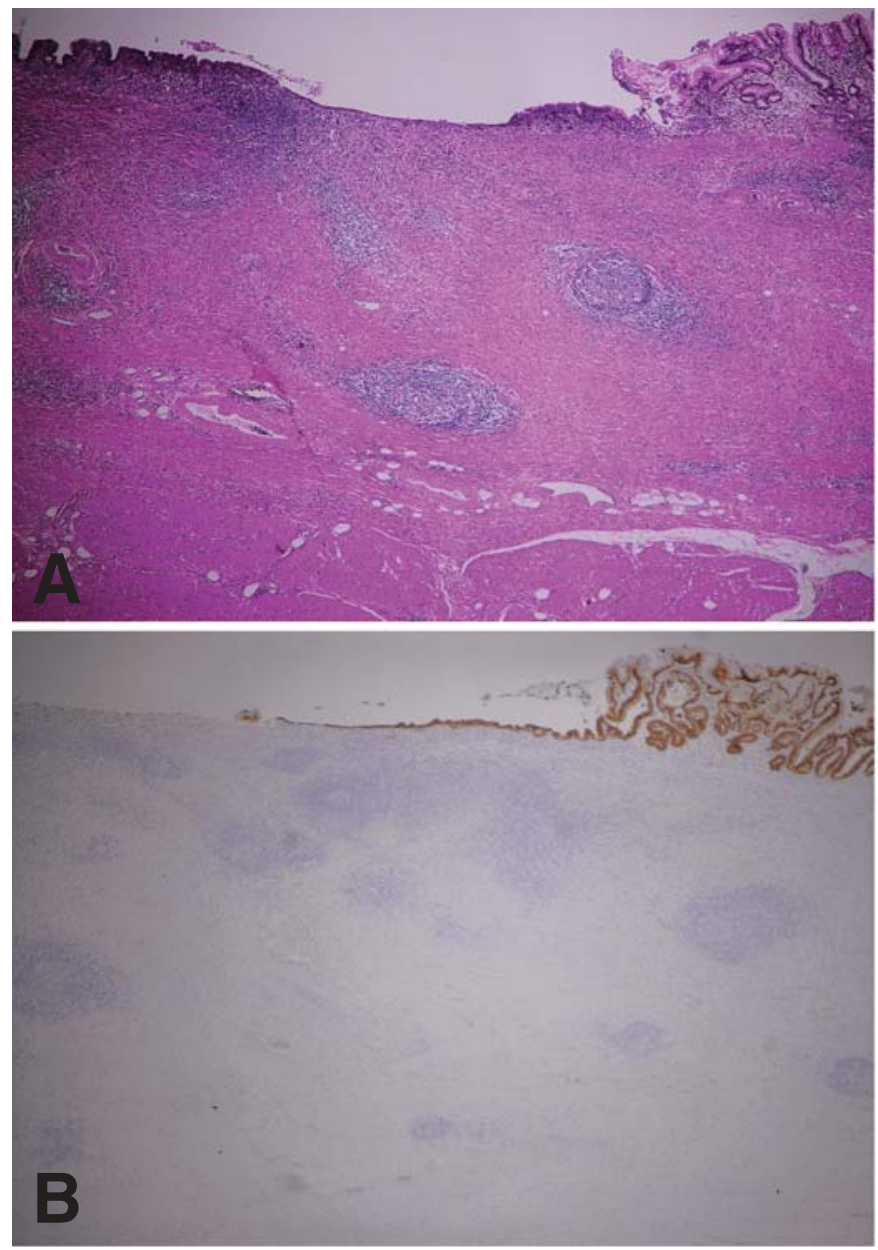

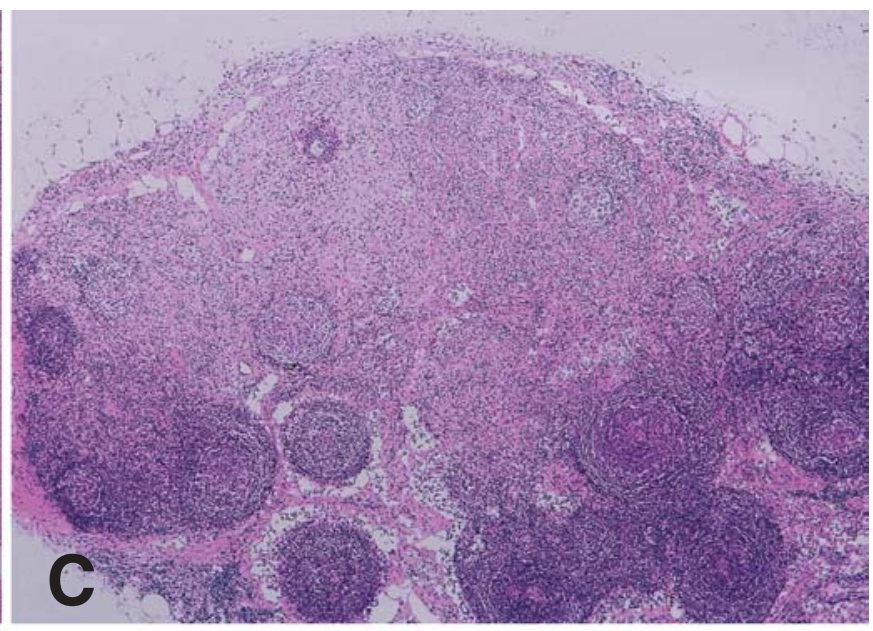

Fig. 3A-C. Microscopic findings of the excised specimen. The lesion was covered with regenerative mucosa, and fibrosis and lymph follicle formation were seen from the submucosa to the subserosa (A). Although negligible gland degeneration was seen in the mucosa, no signet-ring cell remnants were detected by immunostaining for CK22 (B). The assessment of response to the neoadjuvant chemotherapy was a complete response (CR). No lymph node metastasis was found pathologically, but one of the No. 3 lymph nodes showed unnatural fibrosis $(\mathbf{C})$, suggesting degeneration of cancer cells. A H\&E, $\times 2 ; \mathbf{B} \times 2 ; \mathbf{C ~ H} \& \mathrm{E}, \times 4$
S-1 is a novel oral dihydropyrimidine dehydrogenase (DPD) inhibitory fluoropyrimidine (DIF) based on the biochemical modulation of 5-FU [14]. The use of S-1 is recommended in patients with high DPD activity, and measurement of DPD is useful in drug selection $[15,16]$. DPD activity is measured quantitatively by subjecting tumor biopsy specimens to enzyme-linked immunosorbent assay, using an anti-DPD monoclonal antibody [17]. Determination of DPD activity in the specimen may be useful to judge the suitability of neoadjuvant chemotherapy with S-1. Poorly differentiated and anaplastic adenocarcinomas have been shown to have higher levels of DPD activity than welldifferentiated adenocarcinomas. Therefore, S-1 is thought to be more effective in poorly differentiated or anaplastic type lesions [6]. The marked efficacy in the present patient may have been because the primary lesion was relatively small and its histological type was signet-ring cell carcinoma.

Essential conditions for neoadjuvant chemotherapy are low-grade toxicity and high patient compliance. The oral anticancer drug, S-1, which can be used for outpatient therapy, is an ideal drug for neoadjuvant chemotherapy. Further case reports of the administration of S-1 for outpatient neoadjuvant chemotherapy and confirmation of both its safety and effectiveness will, we believe, result in an increase in the prevalence of its use. Observations in the present patient suggest that oral S-1 neoadjuvant chemotherapy may increase the possibility of achieving $\mathrm{CR}$ in advanced gastric cancer therapy.

\section{Conclusion}

Neoadjuvant chemotherapy with oral S-1 for only 2 weeks showed marked efficacy in the treatment of advanced gastric cancer.

Acknowledgments The authors are indebted to Dr. Naoya Hattori for his review of this manuscript. 


\section{References}

1. Sakata Y, Ohtsu A, Hirokoshi N, Sugimachi K, Mitachi Y, Taguchi T. Late phase II study of novel oral fluoropyrimidine anticancer drug S-1 (1 M tegafur- $0.4 \mathrm{M}$ gimestat- $1 \mathrm{M}$ otastat potassium) in advanced gastric cancer patients. Eur J Cancer 1998;34: 1715-20.

2. Koizumi W, Kurihara M, Nakano S, Hasegawa K. Phase II study of S-1, a novel oral derivative of 5-fluorouracil, in advanced gastric cancer. For the S-1 Cooperative Gastric Cancer Study Group. Oncology 2000;58:191-7.

3. Sasaki T. Current topics of S-1 at the 74 th Japanese Gastric Cancer Congress. Gastric Cancer 2003;6 (Suppl):9-12.

4. Kameyama H, Nashimoto A, Yabusaki H, Takii Y, Tsuchiya Y, Tanaka $\mathrm{O}$, et al. A case of advanced gastric cancer responding to neoadjuvant TS-1/CDDP therapy (in Japanese). Gan To Kagaku Ryoho (Jpn J Cancer Chemother) 2003;30:1485-8.

5. Iwahashi M, Nakamori M, Tani M, Yamaue H, Sakaguchi S, Nakamura M, et al. Complete response of highly advanced gastric cancer with peritoneal dissemination after new combined chemotherapy of S-1 and low-dose cisplatin: report of a case. Oncology 2001;61:16-22.

6. Yamamoto Y, Kino Y, Ohara K, Onuma S, Asai K, Kobayashi T, et al. A case report of advanced cardiac cancer showing a complete response to TS-1 as neoadjuvant chemotherapy (in Japanese). Gan To Kagaku Ryoho (Jpn J Cancer Chemother) 2004;31:579-83.

7. Japanese Gastric Cancer Association. Japanese classification of gastric carcinoma. 2nd English ed. Gastric Cancer 1998;1:10-24.

8. Japanese Gastric Cancer Association. Japanese classification of gastric carcinoma. 2nd English edition. Response assessment of chemotherapy and radiotherapy for gastric carcinoma: clinical criteria. Gastric Cancer 2001;4:1-8.

9. Japanese Research Society for Gastric Cancer. The general rules for gastric cancer study 13th ed. (in Japanese). Tokyo: Kanehara; 1999.

10. Shirasaka T, Shimamato Y, Ohshimo H, Yamaguchi M, Kato T, Yonekura K, et al. Development of a novel form of an oral 5fluorouracil derivative (S-1) directed to the potentiation of the tumor selective cytotoxicity of 5-fluorouracil by two biochemical modulators. Anticancer Drugs 1996;7:548-57.

11. Kawai H, Ohtsu A, Boku N, Hamamoto Y, Nagashima F, Muto $\mathrm{M}$, et al. Efficacy and safety profile of S-1 in patients with metastatic gastric cancer in clinical practice: results from a postmarketing survey. Gastric Cancer 2003;6 (Suppl 1):19-23.

12. Japanese Gastric Cancer Association. Gastric cancer treatment guidelines (in Japanese). Tokyo: Kanehara; 2001.

13. Kimura Y, Kikkawa N, Iijima S, Kato T, Naoi Y, Hayashi T, et al. A new regimen for S-1 therapy aiming at adverse reaction mitigation and prolonged medication by introducing a 1-week drug-free interval after each 2-week dosing session: efficacy and feasibility in clinical practice. Gastric Cancer 2003;6 (Suppl 1):34-9.

14. Diasio RB. Clinical implications of dihydropyrimidine dehydrogenase inhibition. Oncology (Williston Park) 1999;13 (7 Suppl 3): $17-21$.

15. Usuki H, Ishimura K, Yachida S, Hagiike M, Okano K, Izuishi K, et al. Dihydropyrimidine dehydrogenase (DPD) activity in gastric cancer tissue and effect of DPD inhibitory fluoropyrimidines. Gastric Cancer 2003;6 (Suppl 1):66-70.

16. Terashima M, Fujiwara H, Takagane A, Abe K, Irinoda T, Gotoh $\mathrm{M}$, et al. Prediction of sensitivity to fluoropyrimidines by metabolic and target enzyme activities in gastric cancer. Gastric Cancer 2003;6 (Suppl 1):71-81.

17. Mori K, Hasegawa M, Nishida M, Toma H, Fukuda M, Kubota T, et al. Expression levels of thymidine phosphorylase and dihydropyrimidine dehydrogenase in various human tumor tissues. Int J Oncol 2000;17:33-8. 